
\title{
3 Research Square \\ CircRNA_0058063 functions as a ceRNA in bladder cancer progression via targeting miR-486- 3p/FOXP4 axis
}

Haote Liang

The First Affiliated Hospital of Wenzhou Medical U

Hang Huang

The First Affiliated Hospital of Wenzhou Medical U

Yeping Li

The First Affiliated Hospital of Wenzhou Medical U

Yongyong Lu

The First Affiliated Hospital of Wenzhou Medical U

Tingyu Ye ( $\square$ wmutingyuye@163.com )

The First Affiliated Hospital of Wenzhou Medical University https://orcid.org/0000-0003-2399-8072

Research article

Keywords: Bladder cancer (BC), circRNA_0058063, miR-486-3p, biomarker, progression

Posted Date: December 9th, 2019

DOI: https://doi.org/10.21203/rs.2.17516/v2

License: (c) (i) This work is licensed under a Creative Commons Attribution 4.0 International License.

Read Full License

Version of Record: A version of this preprint was published at Bioscience Reports on March 1st, 2020. See the published version at https://doi.org/10.1042/BSR20193484. 


\section{Abstract}

Emerging evidences have uncovered critical regulatory roles of circular RNAs (circRNAs) function as dynamic scaffolding molecules in tumorigenesis and progression. However, the aberrant expression and clinical significance of hsa_circ_0058063 (circRNA_0058063) in bladder cancer (BC) remain poorly understood. circRNA expression was analyzed via a microarray in cancerous tissue and non-carcinoma tissues. Luciferase reporter assays and RNA immunoprecipitation (RIP) were both conducted to uncover the function of circRNA_0058063 in BC. circRNA_0058063 was overexpressed in BC tissues compared to adjacent normal tissues. Knockdown of circRNA_0058063 dramatically decreased cell proliferation and invasion, and promoted apoptosis in 5637 and BIU-87 cell lines. Furthermore, mechanistic investigations showed that circRNA_0058063 and FOXP4 could directly bind to miR-486-3p, demonstrating that circRNA_0058063 regulated FOXP4 expression by competitively binding to miR-486-3p. Taken together, circRNA_0058063 functions by sponging miR-486-3p in BC progression, which could be act as a new biomarker and further developed to be a therapeutic target in BC.

\section{Background}

Bladder cancer $(\mathrm{BC})$ was ranked as main factors that are responsible for the cancer-related deaths worldwide, with roughly 74,000 developed cases in the United States in 2015 (Flaig et al., 2017; Sin et al., 2017). The risk of BC increases with age, peaking between 50 and 70 years old, and men are three times higher than women (Smith \& Zaharoff, 2016). The recurrence rate is as high as over 70\% within 5 years, and bladder cancer is required for lifelong cystoscopy (Lorenzatti Hiles et al., 2019).

Circular RNA (circRNA) represent a sort of non-coding RNA, which are widespread presented in mammals (Kristensen, Hansen, Veno, \& Kjems, 2018). circRNAs are formed by RNA splicing, which occurs on a so called "head-to-tail" splicing joint where the receptor splicing site at exon 5 ' ends is linked to the donor site at downstream exon 3' ends. (Y. Zhang et al., 2017). Most circRNAs ( 85\%) resided with known proteincoding genes with structures have intact exons, suggesting that RNA polymerase II (RNA pol II) transcribes them and their biogenesis may be mediated by the splice (Cui et al., 2018; Dong et al., 2017; Zhong et al., 2018) In the past two decades, they have been considered non-functional due to splicing errors. To date, numerous circRNAs have been continuously explored in various cell lines and tissue species (Xu et al., 2018). However, their biological processes and potential functions are poorly understood. In BC, circUBXN7 were identified to suppress cancer viability and metastasis (H. Liu, Chen, et al., 2018). In addition, the potential of circSLC8A1 as a ceRNA to $\mathrm{miR}-130 \mathrm{~b} / \mathrm{miR}-494$ to regulate PTEN expression has been extensively discussed (Lu et al., 2019). However, the mechanistic function of major circRNAs in BC is merely understood. In hepatocellular carcinoma, circTRIM33-12 has thus far been verified to induced tumorigenesis via its action as a buffering molecule for miR-191 (P. F. Zhang et al., 2019). Specifically, in lung cancer circRNA circPRKCl stimulates tumor cell growth through a ceRNA mechanism (X. Zhang, Yang, Zhao, Li, \& Duan, 2019). In pancreatic cancer, circ-LDLRAD3 was found to be significantly associated with lymphatic/venous invasion and metastasis (Yang et al., 2017). Taken together, all these explorations potentiate circRNA's regulatory roles as ceRNA in cancer progression. 
In this research, we reanalyzed the previous microarray datasets and found that circRNA_0058063, is aberrantly expressed in both different BC cell lines and BC carcinoma tissues. Silencing of circRNA_0058063 signaling blocks cell proliferation but promoted cell apoptosis. Furthermore, circRNA_0058063 binds to miR-486-3p and regulates the expression of FOXP4. Therefore, circRNA_0058063 would a potent candidate for future diagnostic and therapeutic purpose of BC.

\section{Methods}

Patient specimens and cell culture

All of 94 cases of BC cancerous tissues and the non-cancerous normal tissues was retrospectively analyzed from the First Affiliated Hospital of Wenzhou Medical University during April 2013 to Oct 2014. BC Cell lines (BIU-87, 5637 and RT-112) were purchased from the American Type Culture Collection (ATCC, Manassas, VA, USA). 293T cell line were donated by Dr Teng of Shanghai Eighth People's Hospital (Shanghai, China). Cells were cultured in DMEM/F12 supplemented with $10 \%$ FBS (Gibco, Grand Island, NY, USA), penicillin (100 $\mu \mathrm{g} / \mathrm{mL}$, Gibco) and streptomycin $\left(100 \mu \mathrm{g} / \mathrm{mL}\right.$, Gibco) at $37^{\circ} \mathrm{C}$ in $95 \%$ humidified air and $5 \% \mathrm{CO} 2$. All studies were conducted by following the protocol provided by the manufacturer. Studies are performed in accordance with the Declaration of Helsinki and adhere to local ethical regulations. We have acquired the written Informed consent from each participant before the initiation of this experimental study. The protocol of the current investigation was authorized by the Institutional Review Board of the First Affiliated Hospital of Wenzhou Medical University.

Microarray analysis

We used microarray datasets for identification of candidate circRNAs at platform GPL19978 (https://www.ncbi.nlm.nih.gov/geo/query/acc.cgi?acc = GSE92675). Four pairs of BC carcinoma tissues and paired para-carcinoma tissues were selected for detection of the expression and potential circRNAs functions.

qRT-PCR

Total RNA was extracted using TRIzol reagent (Invitrogen, USA). Then, cDNA was reverse-transcribed by the Prime Script RT reagent Kit (New England Biolabs, MA, USA) and subjected to RT-PCR measurement using PowerUp SYBR Green Master Mix (Takara Bio, Dalian, China). The relative expressions of each gene were acquired by using the $2-\triangle \triangle C$ t method. The primers were listed below: $5^{\prime}-$

TATGATCCTGTTTGGTGGTCGGCA-3'(forward), 5'-TGGACCAAGATGGGTAGCTTGTGA-3'(reverse) for circRNA_0058063. 5'-CAATGTACGTTCGCTATCGGC-3'(forward), 5'-CTCTCACGCACTTAATGCGAT-3' (reverse) for miR-486-3p. 5'-CGGCACAGCAGCTGAACTTA-3'(forward), 5'GCAACACCTGAAAAAGTGTGA-3'(reverse) for FOXP4. 5'-CTCGCTACACCTCAATACATCG-3'(forward), 5'GCGCCATAAGTCTAGTATTGAGA-3'(reverse) for GAPDH.

Luciferase assay 
siRNA for circRNA_0058063 or scramble siRNA (si-NC) was synthesized by Invitrogen. The miR-486-3p mimic and the negative control (miR-NC) were synthesized by GenePharma (Shanghai, China). Lipofectamine 2000 (Invitrogen, Carlsbad, CA) system was used to conduct cell transfection assay by following the manufacturer's protocol.

\section{CCK8 assay}

As previously described ( $\mathrm{H}$. Liu, Bi, et al., 2018), $2 \times 10^{3}$ cells were split and cultured in 96-well plates at 24 hours before its use for CCK- 8 assay. After 48 hours of transfection, $10 \mu \mathrm{L}$ of CCK-8 solution was then pipetted into the medium. Absorbance was detected at $450 \mathrm{nM}$ on a Spectra Max 250 spectrophotometer (Molecular Devices, USA). After cells were fixed for $15 \mathrm{~min}$ in $4 \%$ of paraformaldehyde, the colonies were stained by $0.05 \%$ crystal violet $(20 \mathrm{~min})$ followed by visualized under a microscope.

\section{Flow cytometry}

The rate of apoptosis of the regulatory cells was examined. Both flow cytometry and annexin $\mathrm{V} /$ propidium iodide staining were applied to detect cell death rate by using the Annexin V-fluorescein isothiocynate Apoptosis Detection Kit (KeyGen, Nanjing, China).

Transwell assay

At the beginning $20 \%$ fetal calf serum was put into the lower chamber, and the conditioned cells were suspended in a medium without FBS and inoculated onto an upper chamber. $48 \mathrm{~h}$ after adding the cells, get rid of the Matrigel in the upper chamber, at the same time the attached cells of invading ability were fixed, and crystal violet was applied to stain the cells, and then it was subjected for imaging with the aid of a microscope.

RNA immunoprecipitation (RIP) assay

With the aid of Lipofectamine 2000 transfection system, $1 \times 10^{7}$ cells were incubated in the RIP buffer transfected with MS2bs-circRNA_0058063mt or MS2bs-circRNA_0058063, and control MS2bs-Rluc. After 48 hours of transfection, RIP was conducted by using the Magna RIP RNA-binding protein immunoprecipitation kit (Millipore). For the RIP assay of Ago2, anti-Ago2 antibody (Millipore) was applied to RIP assay after the transfection for $48 \mathrm{~h}$.

Western blot analysis

First, proteins were subjected to electrophoresis with 10\% SDS-PAGE and then were transferred onto PVDF membrane (Millipore Corp., MA, USA) for $1 \mathrm{hr}$. Then, the membranes were blocked with $5 \%$ non-fat milk prepared by washing buffer for $1 \mathrm{hr}$. Next, the membranes were prepared to be incubated with corresponding antibodies against FOXP4 (1:500, Abcam, USA) to detect the relative protein expression of targets. Anti- $\beta$-actin antibody (1:1000, Affinity, USA) was applied as loading control. After incubating with primary antibody at $4{ }^{\circ} \mathrm{C}$ overnight, the membranes were washed with tris-buffered saline for 4 times and 
each time for 5 minutes. At last, specific corresponding secondary antibodies (1:2000, CST, USA) were further incubated with the membrane at room temperature. Protein bands were visualized and analyzed using Quantity One 4.6.2 software (Bio-Rad, Hercules, CA, USA).

Statistical Analysis

SPSS 21.0 software for Windows (SPSS Inc.) was utilized to conduct statistical analysis. Data sets were expressed as mean \pm s.e.m. The differences between two groups or more were compared by two-tail $t$ test and $\chi 2$ test, respectively. Kaplan-Meier survival curves were analyzed using log-rank tests. High/low circRNA_0058063 expression groups were divided according to the medium expression values. Statistical differences were taken when $\mathrm{P}<0.05$. Triplicate experiments were performed in any of the assay unless otherwise stated.

\section{Results}

circRNA_0058063 is upregulated in BC progression

A total of 312 circRNAs were differentially expressed (Fold change $>2, P<0.05$ ) with the analysis of circRNA microarray from GEO database. 195 circRNAs were up-regulated, and 117 circRNAs were significantly down-regulated in four bladder cancer samples. The top dysregulated circRNAs were presented in the heat map (Fig. 1A). Our results indicated that circRNA_0058063's expression level was augmented in both BC cell lines (Fig. 1B) and the tissues (Fig. 1C). Kaplan-Meier analysis showed 94 cases of BC patients with the higher expression level of circRNA_0058063 had a relatively low 5-year OS, indicating that circRNA_0058063 expression was negatively correlated with carcinogenesis in individual BC patients (Fig. 1D). And it was further confirmed that higher circRNA_0058063 expression contributes to advanced stage and metastasis of the disease (Table 1). Taken together, it potentiates circRNA_0058063's critical role in the regulation of BC.

circRNA_0058063 knockdown suppressed cell proliferation and invasion

Since the expression of circRNA_0058063 was induced in BC, the expression of circRNA_0058063 was silenced in BC to determine the regulatory events. As shown in fig. 2A, silencing of circRNA_0058063 was successfully achieved. Moreover, silencing of circRNA_0058063 inhibit the proliferation rate of 5637 and BIU-87 cell lines (Fig. 2B). Transwell assay showed inhibition of cyclin invasion by circRNA_0058063 (Fig. 2C). Apoptosis assays indicated that inhibition of circRNA_0058063 contributed to elevated cell apoptosis (Fig. 2D). Therefore, it would be interesting to conclude that silencing of circRNA_0058063 inhibits both 5637 and BIU-87 cell proliferation and cell invasion, yet promoted apoptosis of 5637 and BIU-87 cell lines during BC progress in vitro.

circRNA_0058063 acts as a sponge for miR-486-3p

The intracellular residence of circRNA_0058063 was further explored and it was revealed that circRNA_0058063 is mainly distributed in the cytoplasm (Fig. 3A), suggesting that circRNA_0058063 may 
act as a miRNA sponge to induce miRNAs. Based on the results, the Circular RNA Interactome (https://circinteractome.nia.nih.gov/index.html) was applied to study the underlying mechanism of circRNA/miRNA interactions and we found the complementary site of miR-486-3p in the circRNA_0058063 sequence. (Fig. 3B). Furthermore, miR-486-3p expression was mitigated in 5637 and BIU-87 cell lines and the BC tissues (Fig. 3C-D). Next, the direct binding relationship between miR-486$3 p$ and circRNA_0058063 was confirmed with a luciferase assay. When luciferase reporter gene and the miR-486-3p mimic were co-transfected into the 5637 and BIU-87 cell lines, the luciferase intensities were greatly reduced (Fig. 3E). To elucidate whether the binding of circRNA_0058063 and miR-486-3p was direct or indirect, a RIP assay was conducted. It was indicated that miR-486-3p was predominantly resided at the site of MS2bs-circRNA_0058063, potentiating a direct binding of circRNA_0058063 and miR-486-3p (Fig. 3F). In summary, our data confirm that circRNA_0058063 could directly inhibit the $\mathrm{miR}-486-3 p$ expression by directly combining to the binding site.

circRNA_0058063 regulates FOXP4 by miR-486-3p

To investigate whether circRNA_0058063 modifies miR-486-3p to regulate target gene expression, we predicted that FOXP4 is its regulatory downstream by using TargetScan to evaluate the downstream gene of miR-486-3p (Fig. 4A). We detected FOXP4 expression levels and it was revealed that the expression of FOXP4 was increased in the BC cell line (Fig. 4B) and the tissues of BC (Fig. 4C). Next, the luciferase reporter assay suggested that luciferase activity of FOXP4 was significantly decreased by the miR-486$3 p$ mimic and the luciferase reporter gene (Fig. 4D). miR-486-3p inhibited the expression of FOXP4 (Fig. 4E). Next, the RIP assay for Ago2 showed circRNA_0058063, FOXP4 and miR-486-3p were mainly enriched to Ago2 (Fig. 4F). This result indicated that circRNA_0058063 and FOXP4 were subjected to Ago2-related RISC, and then it promoted their interaction with miR-486-3p. Furthermore, inhibition of circRNA_0058063 lowered the recruitment of Ago2 to circRNA_0058063, but elevated the recruitment of Ago2 enrichment to FOXP4 (Fig. 4G), which revealed that circRNA_0058063 can act as a ceRNA and antagonize with FOXP4 to bind with miRNA. In addition, the expression of FOXP4 was detected when circRNA_0058063 was silenced and it was found that FOXP4's expression was significantly reduced, but it was significantly recersed by miR-486-3p LNA (Fig. 4H). Taken together, circRNA_0058063 tightly regulated the expression of FOXP4 as a ceRNA by inducing miR-486-3p.

\section{Discussion}

An abundant of researches have explored circRNA's crucial functions in the occurrence and progression of diverse cancers (J. Liu, Liu, Wang, \& He, 2017; Qin et al., 2018). In lung cancer, the regulatory roles of circRNA has been uncovered in various cell biological processes (Smid et al., 2019). During the progression of glioma, circPTN blocked cancer growth and metastasis (Chen et al., 2019). Meanwhile, the exploration of circRNAs in $\mathrm{BC}$ is rarely existed. Therefore, we utilized the circRNA expression microarray assay in BC and indicated that circRNA_0058063 was up-regulated in BC. Hsa_circ_0058063 is located at the domain of chr2: 216177220-216213972 according to the human reference genome (GRCh38.p13) and is thought to originate from the gene ATIC, which residues at chromosome $2 q 35$. Therefore, 
hsa_circ_0058063 was named as "circRNA_0058063". Further investigations demonstrated that silencing of circRNA_0058063 blocked cell proliferation and cell invasion, but promoted cell death, suggesting that circRNA_0058063 can modulate BC progression. Therefore, circRNA_0058063 potentiates its potential as target for pathological detection and treatment.

The reported researches have indicated that circRNA regulates gene expression through cavernous miRNAs. In colorectal cancer, CCDC66 promotes cancer growth and metastasis through targeting miRNA-33b and miR-93 (Hsiao et al., 2017). Bi et al. proved that circRNA_102171 regulates cell proliferation and apoptosis, and causes malignant transformation by blocking CTNNBIP1 in papillary thyroid carcinoma (Bi et al., 2018). Here, we found that circRNA_0058063 directly bound with miR-486$3 p$ and worked as a miRNA sponge. Furthermore, we found that FOXP4 is the target gene of miR-486$3 p$. It was reported that microtubule-associated proteins (MAPs) mainly reside in the cytoplasmic fraction and bind with tubulin and facilitate their aggregation (Devanna, Middelbeek, \& Vernes, 2014). Others have suggested MAP's involvement in the control of mitochondrial balance or apoptosis (Hu et al., 2014). Previous studies have reported that FOXP4 overexpresses and regulates tumor cell growth in non-small cell lung cancer (Wu, Xiao, Zhou, Zhou, \& Yan, 2019). FOXP4 has been certified in the progression of prostate cancer (Hicks, Miele, Koganti, \& Vijayakumar, 2013). In the present study, the abundance of FOXP4 was found to be elevated in BC. Further exploration suggested circRNA_0058063 acts as a bait for miR-486-3p to modulate FOXP4 expression. Inhibition of circRNA_0058063 decreased FOXP4's expression, which was overturned by silencing of miR-486-3p.

\section{Conclusion}

To sum up, the expression of circRNA_0058063 was elevated in BC. Silencing of circRNA_0058063 blockeds cell proliferation and cell invasion, however promoted cell death. Mechanistically, circRNA_0058063 regulates the expression of FOXP4 by the sponge miR-486-3p. circRNA_0058063 potentiates as a valuable candidate for the early detection and prognosis of $\mathrm{BC}$.

\section{Declarations}

\section{Abbreviations}

Bladder cancer (BC), circular RNAs (circRNAs), RNA polymerase II (RNA pol II),

\section{Ethics approval and consent to participate}

The protocol of the current investigation was authorized by the Institutional Review Board of the First Affiliated Hospital of Wenzhou Medical University. Written informed consent was obtained from all the participants in the study. 


\section{Consent to publish}

Not applicable.

\section{Availability of data and materials}

The authors declare that the data supporting the findings of this study are available within the article.

\section{Competing interests}

The authors declare that they have no competing interests.

\section{Funding}

NA.

Haote Liang MM, Hang Huang MD, Yeping Li MD, Yongyong Lu MD, Tingyu Ye MD§

\section{Authors' Contributions}

Conception and intellectual input: YTY; designed and performance of experimentation: LHT; data collection and manuscript drafting: HH, LYP. Statistical analyses and data interpretation: LYY; All authors read and approved the final manuscript.

\section{Acknowledgements}

NA.

\section{References}

1. Bi W, Huang J, Nie C, Liu B, He G, Han J, Pang R, Ding Z, Xu J, Zhang J (2018). CircRNA circRNA_102171 promotes papillary thyroid cancer progression through modulating CTNNBIP1-dependent activation of betacatenin pathway. J Exp Clin Cancer Res 37: 275. doi: 10.1186/s13046018-0936-7

2. Chen J, Chen T, Zhu Y, Li Y, Zhang Y, Wang Y, Li X, Xie X, Wang J, Huang $\mathrm{M}$ et al. (2019). circPTN sponges miR-145-5p/miR-330-5p to 
promote proliferation and stemness in glioma. J Exp Clin Cancer Res 38: 398. doi: 10.1186/s13046-019-1376-8

3. Cui X, Wang J, Guo Z, Li M, Li M, Liu S, Liu H, Li W, Yin X, Tao J et al. (2018). Emerging function and potential diagnostic value of circular RNAs in cancer. Mol Cancer 17: 123. doi: 10.1186/s12943-018-0877-y

4. Devanna P, Middelbeek J, Vernes SC (2014). FOXP2 drives neuronal differentiation by interacting with retinoic acid signaling pathways. Front Cell Neurosci 8: 305. doi: 10.3389/fncel.2014.00305

5. Dong Y, He D, Peng Z, Peng W, Shi W, Wang J, Li B, Zhang C, Duan C (2017). Circular RNAs in cancer: an emerging key player. J Hematol Oncol 10: 2. doi: 10.1186/s13045-016-0370-2

6. Flaig TW, Kamat AM, Hansel D, Ingersoll MA, Barton Grossman H, Mendelsohn C, DeGraff D, Liao JC, Taylor JA, 3rd (2017). Proceedings of the 3rd Annual Albert Institute for Bladder Cancer Research Symposium. Bladder Cancer 3: 211-223. doi: 10.3233/BLC-170111

7. Hicks C, Miele L, Koganti T, Vijayakumar S (2013). Comprehensive assessment and network analysis of the emerging genetic susceptibility landscape of prostate cancer. Cancer Inform 12: 175-191. doi: 10.4137/CIN.S12128

8. Hsiao KY, Lin YC, Gupta SK, Chang N, Yen L, Sun HS, Tsai SJ (2017). Noncoding Effects of Circular RNA CCDC66 Promote Colon Cancer Growth and Metastasis. Cancer Res 77: 2339-2350. doi: 10.1158/00085472.CAN-16-1883

9. Hu J, Chu Z, Han J, Zhang Q, Zhang D, Dang Y, Ren J, Chan HC, Zhang J, Huang Y (2014). Phosphorylation-dependent mitochondrial translocation of MAP4 is an early step in hypoxia-induced apoptosis in cardiomyocytes. Cell Death Dis 5: e1424. doi: 10.1038/cddis.2014.369

10. Kristensen LS, Hansen TB, Veno MT, Kjems J (2018). Circular RNAs in cancer: opportunities and challenges in the field. Oncogene 37: 555-565. doi: 10.1038/onc.2017.361

11. Liu H, Bi J, Dong W, Yang M, Shi J, Jiang N, Lin T, Huang J (2018). Invasion-related circular RNA circFNDC3B inhibits bladder cancer progression through the miR-1178-3p/G3BP2/SRC/FAK axis. Mol Cancer 17: 161. doi: 10.1186/s12943-018-0908-8 
12. Liu H, Chen D, Bi J, Han J, Yang M, Dong W, Lin T, Huang J (2018). Circular RNA circUBXN7 represses cell growth and invasion by sponging miR-1247-3p to enhance B4GALT3 expression in bladder cancer. Aging (Albany NY) 10: 2606-2623. doi: 10.18632/aging.101573

13. Liu J, Liu T, Wang X, He A (2017). Circles reshaping the RNA world: from waste to treasure. Mol Cancer 16: 58. doi: 10.1186/s12943-017-0630-y

14. Lorenzatti Hiles G, Cates AL, El-Sawy L, Day KC, Broses LJ, Han AL, Briggs HL, Emamdjomeh A, Chou A, Abel EV et al. (2019). A surgical orthotopic approach for studying the invasive progression of human bladder cancer. Nat Protoc 14: 738-755. doi: 10.1038/s41596-018-0112-8

15. Lu Q, Liu T, Feng H, Yang R, Zhao X, Chen W, Jiang B, Qin H, Guo X, Liu $\mathrm{M}$ et al. (2019). Circular RNA circSLC8A1 acts as a sponge of miR130b/miR-494 in suppressing bladder cancer progression via regulating PTEN. Mol Cancer 18: 111. doi: 10.1186/s12943-019-1040-0

16. Qin C, Liu CB, Yang DG, Gao F, Zhang X, Zhang C, Du LJ, Yang ML, Li JJ (2018). Circular RNA Expression Alteration and Bioinformatics Analysis in Rats After Traumatic Spinal Cord Injury. Front Mol Neurosci 11: 497. doi: 10.3389/fnmol.2018.00497

17. Sin MLY, Mach KE, Sinha R, Wu F, Trivedi DR, Altobelli E, Jensen KC, Sahoo D, Lu Y, Liao JC (2017). Deep Sequencing of Urinary RNAs for Bladder Cancer Molecular Diagnostics. Clin Cancer Res 23: 3700-3710. doi: 10.1158/1078-0432.CCR-16-2610

18. Smid M, Wilting SM, Uhr K, Rodriguez-Gonzalez FG, de Weerd V, PragerVan der Smissen WJC, van der Vlugt-Daane M, van Galen A, Nik-Zainal S, Butler A et al. (2019). The circular RNome of primary breast cancer. Genome Res 29: 356-366. doi: 10.1101/gr.238121.118

19. Smith SG, Zaharoff DA (2016). Future directions in bladder cancer immunotherapy: towards adaptive immunity. Immunotherapy 8: 351-365. doi: $10.2217 /$ imt.15.122

20. Wu X, Xiao Y, Zhou Y, Zhou Z, Yan W (2019). LncRNA FOXP4-AS1 is activated by PAX5 and promotes the growth of prostate cancer by sequestering miR-3184-5p to upregulate FOXP4. Cell Death Dis 10: 472. doi: 10.1038/s41419-019-1699-6 
21. Xu C, Haque F, Jasinski DL, Binzel DW, Shu D, Guo P (2018). Favorable biodistribution, specific targeting and conditional endosomal escape of RNA nanoparticles in cancer therapy. Cancer Lett 414: 57-70. doi: 10.1016/j.canlet.2017.09.043

22. Yang F, Liu DY, Guo JT, Ge N, Zhu P, Liu X, Wang S, Wang GX, Sun SY (2017). Circular RNA circ-LDLRAD3 as a biomarker in diagnosis of pancreatic cancer. World J Gastroenterol 23: 8345-8354. doi:

10.3748/wjg.v23.i47.8345

23. Zhang PF, Wei CY, Huang XY, Peng R, Yang X, Lu JC, Zhang C, Gao C, Cai JB, Gao PT et al. (2019). Circular RNA circTRIM33-12 acts as the sponge of MicroRNA-191 to suppress hepatocellular carcinoma progression. Mol Cancer 18: 105. doi: 10.1186/s12943-019-1031-1

24. Zhang X, Yang H, Zhao L, Li G, Duan Y (2019). Circular RNA PRKCI promotes glioma cell progression by inhibiting microRNA-545. Cell Death Dis 10: 616. doi: 10.1038/s41419-019-1863-z

25. Zhang Y, Liang W, Zhang P, Chen J, Qian H, Zhang X, Xu W (2017). Circular RNAs: emerging cancer biomarkers and targets. J Exp Clin Cancer Res 36: 152. doi: 10.1186/s13046-017-0624-z

26. Zhong Y, Du Y, Yang X, Mo Y, Fan C, Xiong F, Ren D, Ye X, Li C, Wang Y et al. (2018). Circular RNAs function as ceRNAs to regulate and control human cancer progression. Mol Cancer 17: 79. doi: 10.1186/s12943-0180827-8

\section{Table}

Table 1. Association between circRNA_0058063 expression and different clinicopathological characteristics of $\mathrm{BC}$ patients 


\begin{tabular}{|c|c|c|c|}
\hline Variable & $n$ & Relative circRNA_0058063 level & $P$ value \\
\hline Gender & & & \\
\hline Female & 44 & $5.24 \pm 1.27$ & \\
\hline Male & 50 & $5.51 \pm 1.48$ & 0.721 \\
\hline Age (years) & & & \\
\hline $\begin{array}{l}<60 \\
\geq 60\end{array}$ & $\begin{array}{l}58 \\
36\end{array}$ & $\begin{array}{l}5.12 \pm 1.36 \\
5.64 \pm 1.41\end{array}$ & 0.135 \\
\hline Number of tumors & & & \\
\hline $\begin{array}{l}\text { Single } \\
\text { Multiple }\end{array}$ & $\begin{array}{l}71 \\
23\end{array}$ & $\begin{array}{l}4.89 \pm 1.32 \\
5.84+1.71\end{array}$ & 0118 \\
\hline Stage & & & \\
\hline $\begin{array}{l}\mathrm{T} 0 / \mathrm{T} 1 \\
\geq \mathrm{T} 2\end{array}$ & $\begin{array}{l}80 \\
14\end{array}$ & $\begin{array}{l}4.13 \pm 1.26 \\
8.28 \pm 1.47\end{array}$ & 0.003 \\
\hline Grade & & & \\
\hline $\begin{array}{l}\mathrm{G} 1 / 2 \\
\mathrm{G} 3\end{array}$ & $\begin{array}{l}37 \\
57 \\
\end{array}$ & $\begin{array}{l}3.51 \pm 1.23 \\
7.97 \pm 1.45 \\
\end{array}$ & 0.004 \\
\hline
\end{tabular}

